\title{
Fault Detection, Estimation/Reconstruction, and Fault-Tolerant Control: Theory and Practice
}

\author{
Wen Chen, ${ }^{1}$ Afef Fekih, ${ }^{2}$ and Zehui Mao ${ }^{3}$ \\ ${ }^{1}$ Division of Engineering Technology, Wayne State University, Detroit, MI 48201, USA \\ ${ }^{2}$ Department of Electrical and Computer Engineering, University of Louisiana at Lafayette, Lafayette, LA 70504, USA \\ ${ }^{3}$ College of Automation Engineering, Nanjing University of Aeronautics and Astronautics, Nanjing 211106, China
}

Correspondence should be addressed to Wen Chen; wchenc@wayne.edu

Received 11 July 2016; Accepted 11 July 2016

Copyright (C) 2016 Wen Chen et al. This is an open access article distributed under the Creative Commons Attribution License, which permits unrestricted use, distribution, and reproduction in any medium, provided the original work is properly cited.

\section{Introduction}

The increasing complexity of modern engineering systems will correspondingly increase the possibility of system faults and/or failures. The occurrence of sensor, actuator, and/or component failures may dramatically degrade system performance and even result in catastrophic system collapse. As a response to high requirement for system safety, reliability, and survivability, fault diagnosis and fault-tolerant control (FTC) for dynamic systems have been attractive subjects of many investigations in control community and have received considerable attention during the past few decades, and a great deal of research progress has been made.

The articles in this special issue speak on recent development of fault detection, estimation/reconstruction, and faulttolerant control as well as their applications. The following sections briefly explain the outcome of published articles in this special issue. They are divided into five key research areas based on publications.

\section{Fault Localization}

W. Zheng et al. proposed a fault localization method based on a deep neural network (DNN). It is capable of achieving the complex function approximation and attaining distributed representation for input data by learning a deep nonlinear network structure. Another advantage is its strong ability to learn from a small-sized training dataset. The DNN-based model is trained utilizing the coverage data and the results of test cases as inputs and the faults can be located by testing the trained model using the virtual test suite. Another article on fault localization is proposed by N. Li et al., which is about building up a balanced test suite for spectrum-based fault localization. They proposed a PNF strategy to reduce test suite and build up a more balanced one for SBFL. They have evaluated the strategy by experiments using the Siemens and Space program. Experiments indicated that the proposed PNF strategy can be used to construct a new test suite effectively. Compared with the original test suite, the new one has a smaller size and more balanced ratio of failed test cases to previous test cases, while it has the same statement coverage and fault localization accuracy.

\section{Fault-Tolerant Control}

S. Wang et al. suggested a fault-tolerant control scheme for autopilot of a small fixed-wing UAV. The flight control system is designed based on an open-source autopilot (Pixhawk). Real-time kinematic (RTK) GPS is introduced due to its high accuracy. Some modifications on the longitudinal and lateral guidance laws are achieved to improve the flight control performance. Moreover, a data fusion-based fault-tolerant control scheme is integrated into altitude control and speed control for altitude and airspeed sensor failures. Actual flight test results show that the fixed-wing UAV can track the desired altitude and speed commands during the whole flight process by the fault-tolerant control-based autopilot. 


\section{Fault Diagnosis Methods}

New fault-diagnostic methods have been presented using different techniques such as observers, filters, SROS-ELM, and the indiscernibility dynamic kernel PCA. D. Krokavec et al. suggested a new fault detection filter design that relaxes the existing conditions by adapting the unitary system principle in approximation of fault detection filter transfer function matrix for continuous-time linear MIMO systems. H. M. Tran and H. Trinh examined the design of minimal-order residual generators for detecting and isolating actuator and/or component faults in dynamical systems. First, existence conditions and design residual generators are derived using only first-order observers to detect and identify the faults. When the first-order functional observers do not exist, then based on a parametric approach to the solution of a generalized Sylvester matrix equation a systematic procedure for designing residual generators utilizing minimal-order functional observers is developed. J. Zhou et al. proposed an analytical redundancy design for aeroengine sensor fault diagnostics based on SROS-ELM. The modified online sequential extreme learning machine is employed to train the model online and estimate sensor measurements. It selectively updates the output weights of neural networks, tackles the problems of singularity and ill-posedness by regularization, and adopts a dual activation function in the hidden nodes. Another article related to a new method for diagnosing faults of rolling bearing based on a dual-tree complex wavelet packet transform and improved multiscale permutation entropy is presented by G. Tang et al. The experimental data analysis results have verified the effectiveness of the presented diagnosis method and demonstrated that this method can be applied to distinguish the different fault types and fault degrees of rolling bearings. Aimed at complicated faults detection of distillation column industrial processes, Q. Gao et al. suggested a new indiscernibility dynamic kernel principal component analysis (I-DKPCA) method. Compared with traditional statistical techniques, the I-DKPCA can not only capture nonlinear property and dynamic characteristic of processes, but also extract relevant items from all of the variables.

\section{Safety Assessment}

L. Meng et al. pursued a safety assessment for electrical motor based on an SOM neural network. In this work, the system's health clustering and safety identification are carried out. With the switch devices' faults data obtained from the dSPACE simulation platform, the health assessment algorithm is proposed and verified. The results show that the algorithm can achieve the system's fault diagnosis and health assessment.

\section{Fault Analysis}

B. Li and M. Li calculated and analyzed the effect of the permanent magnet eddy current loss fault due to magnet segmentation. A three-dimensional finite-element model was firstly established; then, model meshes and boundary conditions were handled specially, and permanent magnet eddy current loss fault was calculated and analyzed. Finally, calculated results were compared and explained.

Wen Chen Afef Fekih

Zehui Mao 


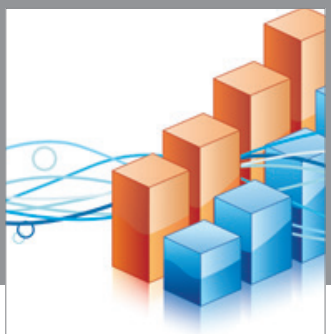

Advances in

Operations Research

vatem alat4

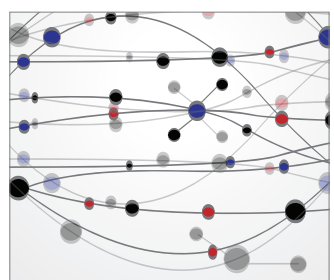

\section{The Scientific} World Journal
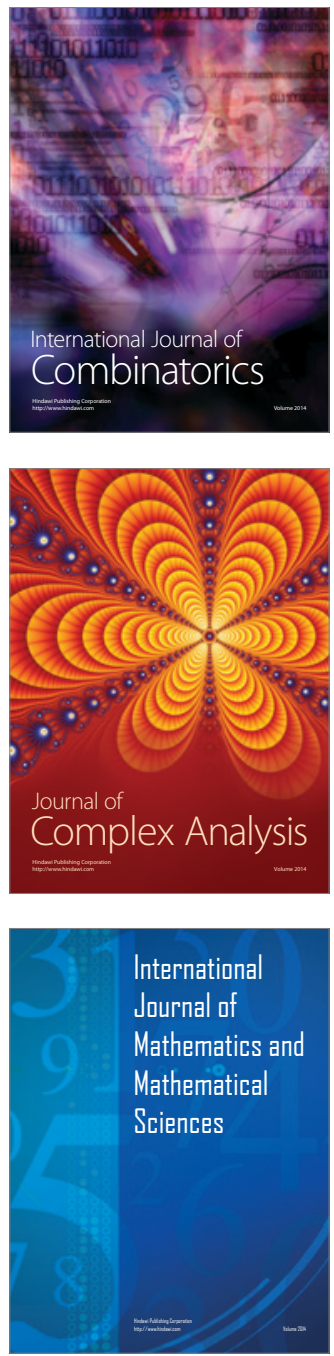
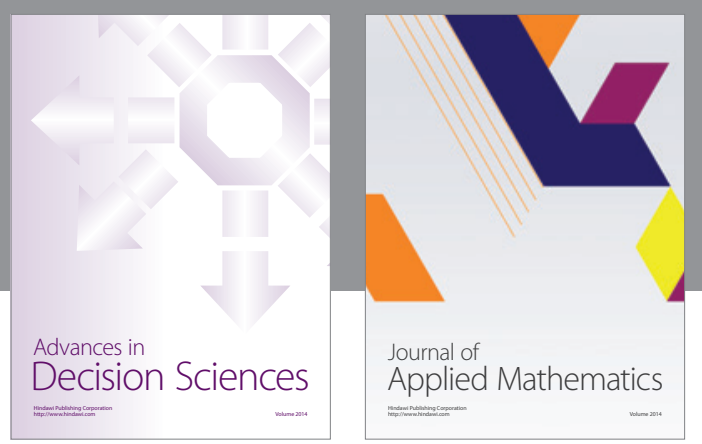

Algebra

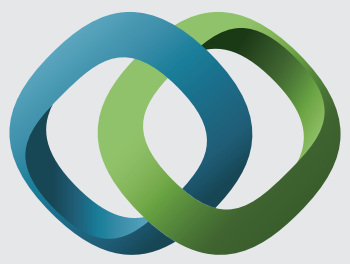

\section{Hindawi}

Submit your manuscripts at

http://www.hindawi.com
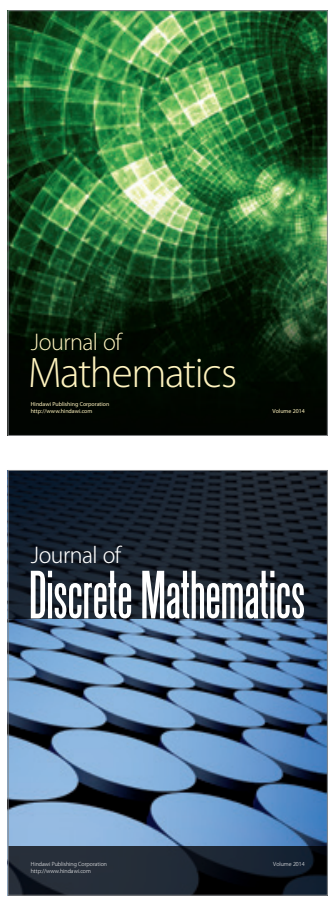

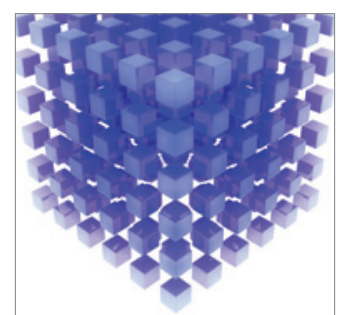

Mathematical Problems in Engineering
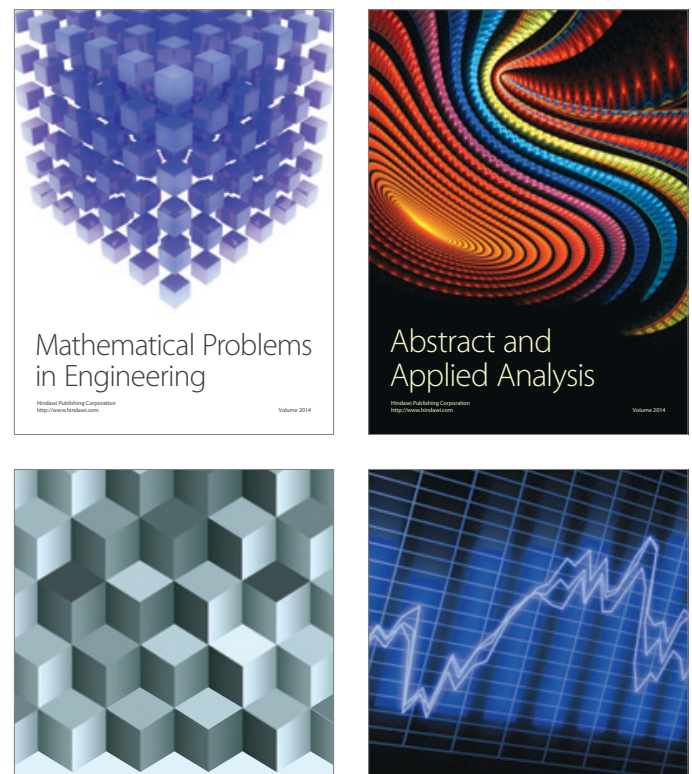

Journal of

Function Spaces

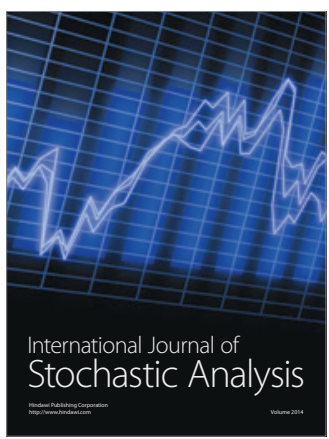

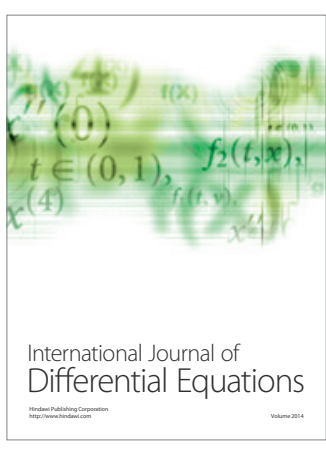
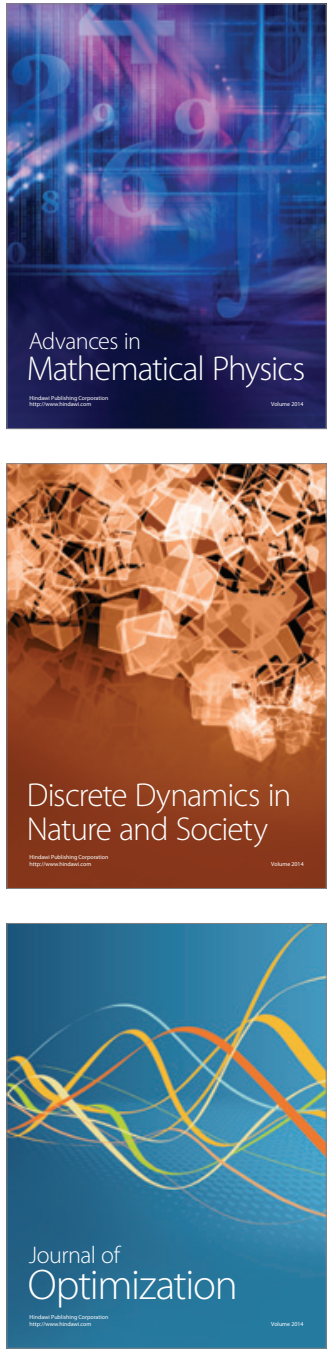